Dr. med. Dirk Einecke Chefredakteur dirk.einecke@springer.com

\author{
Blutdruck als Prädiktor
}

\section{US-Forscher: „Der plötzliche Herztod ist vermeidbar!“}

Wer sich sorgt, er könne irgendwann am plötzlichen Herztod (PHT) sterben, sollte vor allem seinen Blutdruck im Auge behalten. Dieser war in einer Kohortenstudie auf

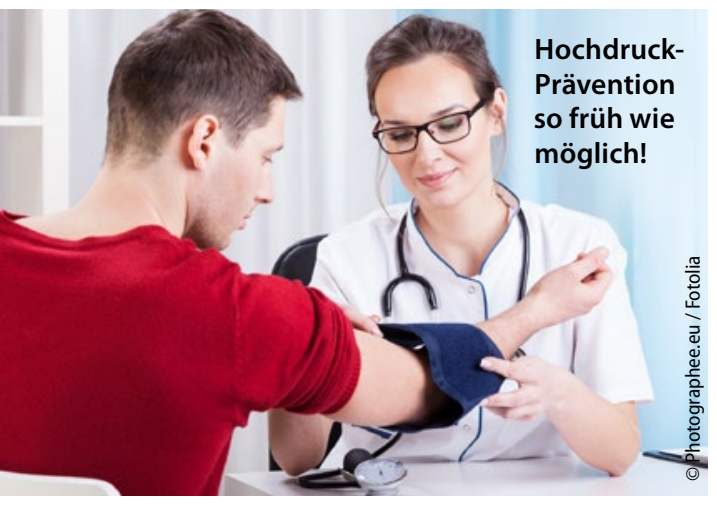

\section{Kutanes Plattenepithelkarzinom}

\section{Weißweintrinker sollten auf ihre Haut achten}

Weißweintrinker haben ein erhöhtes Risiko für ein kutanes Plattenepithelkarzinom (SCC): Die Teilnehmer der Nurses' Health Study (NHS), der NHS II sowie der Health Professionals' Follow-up Study hatten Auskunft über ihre Trinkgewohnheiten gegeben. Innerhalb von 19 Jahren wurden 2.938
Basis von Framingham-Daten der beste Prädiktor für das PHT-Lebenszeitrisiko. Daten von 2.294 Männern und 2.785 Frauen flossen in die Studie ein. In 160.396 Personenjahren kam es zu 375 PHT-Fällen.

Männer waren deutlich stärker gefährdet als Frauen. Die schlechtesten Karten hatten Männer, die schon mit 45 mindestens zwei kardiovaskuläre Risikofaktoren aufwiesen. In diesem Fall betrug das kumulative PHT-Risiko über 12\%. Besser als jeder andere Einzelparameter sagte der Blutdruck das Risiko voraus. Ein 45-Jähriger mit einem Blutdruck unter 120/80 mmHg hatte nur ein 5,5\%iges Risiko, bei Werten über 160/100 mmHg lag es bei 16,3\%.

- JAm Heart Assoc. 2016;5:e002398

\section{Zu viele Antibiotika}

\section{Clostridien im Pflegeheim}

Bewohner von Pflegeheimen sind stark gefährdet, sich mit Clostridium difficile zu infizieren. Dies zeigt eine retrospektive Studie mit Daten von über 6.000 US-Veteranen, die in verschiedenen Pflegeeinrichtungen versorgt werden.

Die Inzidenz der Clostridieninfektionen bei Langzeitpflege betrug danach 3,7 pro 10.000 Tage Heimaufenthalt. Sowohl ein hoher Antibiotikagebrauch als auch die Einschleppung der Keime durch Rückkehrer aus Akutkrankenhäusern waren signifikant mit einer $\mathrm{Zu}$ nahme der Clostridieninzidenzen bei den Heimbewohnern assoziiert.

- Ann Intern Med 2016;164:787-94

\section{Atemwegsinfekte}

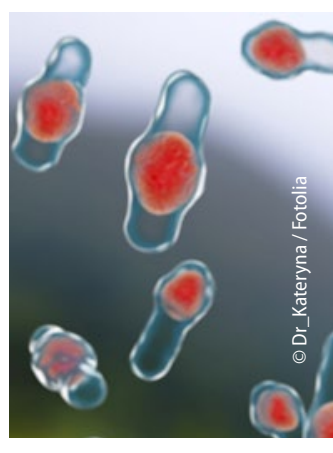

\section{Kaum Komplikationen durch Antibiotikaverzicht} ziert. Dabei zeigte sich eine dosisabhängige Assoziation zum Weißweinkonsum. Wer fünf oder mehr Gläser pro Woche davon trank, hatte ein um 31\% höheres Hautkrebsrisiko als ein Nichttrinker.

- Nutrition and Cancer 2016;68(4):545-553

\title{
Britische Analyse
}

\section{Frühlingskinder haben höchstes MS-Risiko}

Ob jemand an MS erkrankt, hängt auch von seinem Geburtszeitpunkt ab. Wissenschaftler aus Oxford analysierten Geburtsdaten von über 21.000 MS-Patienten, die zwischen 1938 und 1980 geboren wurden. Nach ihren Berechnungen ist bei Briten die MS-Rate um 17\% höher, wenn sie im April statt im November geboren wurden, für die Jahrgänge 1965 bis 1980 liegt der Unterschied sogar bei 24\%. Jahreszeitlich schwankende Vitamin-D-Spiegel oder winterliche Infekte der Mutter werden als mögliche Ursachen des Phänomens diskutiert.

- JAMA Neurol 2016; epub June 27, 2016.
Der Rückgang von Antibiotikaverordnungen gegen selbstlimitierende Atemwegsinfekte ist nicht mit einer relevanten Zunahme von Komplikationen verbunden, so das Ergebnis einer englischen Studie. Ausgewertet wurden Daten von 610 Allgemeinarztpraxen mit mehr als 4 Millionen $\mathrm{Pa}$ tienten. In den 25\% der Praxen mit den wenigsten Antibiotikaverordnungen (bei $38 \%$ aller Atemwegsinfekte) waren im Vergleich zu dem Viertel mit den höchsten Verschreibungsraten (65\%) etwas mehr Pneumonien und Peritonsillarabszesse zu verzeichnen. Bei anderen Komplikationen zeigte sich kein Zusammenhang mit der Häufigkeit von Antibiotikaverordnungen. - BMJ 2016;354:i3410 